\title{
Editorial
}

\section{Reemerging Ebola haemorrhagic fever 2014 and the risks of its introduction into Brazil}

The Ebola virus was identified in 1976 during epidemics of haemorrhagic fever in southern Sudan and northern Zaire (now the Democratic Republic of the Congo). The average mortality rate of Ebola virus disease, formerly known as Ebola haemorrhagic fever, is around 50\% with rates varying from $25-90 \%$ in past outbreaks. The chronology of the outbreaks of the disease in different countries since 1976, according to the virus subtypes, numbers of cases and notified deaths up to November 2014 can be seen in Table. In this latest epidemic, eight countries have been affected: Guinea, Liberia and Sierra Leone have been the most affected countries while cases have been recorded in Nigeria, Senegal, United States of America (USA), Spain and Mali. A separate, unrelated Ebola outbreak occurred in Boende, Equateur, an isolated part of the Democratic Republic of Congo in August-October 2014.

Filoviral diseases are caused by viruses of the family Filoviridae, which is composed by the Marburg, Cueva and Ebola viruses. Filoviruses are particles formed by long filaments that contain a single strand of RNA and they cause haemorrhagic fever in humans and non-human primates (monkeys, gorillas and chimpanzees). Given the lethal nature of these viruses, they are considered to be class IV pathogens, i.e., highly dangerous. Five subtypes of the Ebola virus have been identified: Zaire Ebola virus, Reston Ebola virus, Sudan Ebola virus, Tai Forest Ebola virus and Bundibugyo Ebola virus (Figure). The Zaire, Sudan and Bundibugyo subtypes have been responsible for major outbreaks of the disease. On the other hand, although the Reston and Tai Forest subtypes may affect human beings, no cases of fever and death among humans have been notified.

The hosts of the Ebola virus are fruit-eating bats of the family Pteropodidae. Introduction of the virus into the human population occurs through close contact with blood, secretions, organs and other fluids from infected animals such as chimpanzees, gorillas, fruit-eating bats, monkeys, wild antelopes and wild pigs that are found sick or dead in the rainforests. The Ebola virus is transmitted among human beings through direct contact by means of lacerated skin or mucosa, with blood, secretions, organs and other body fluids from infected individuals or by means of materials and clothing that have become contaminated with these fluids.

Healthcare professionals (physicians, nurses, pathologists and researchers) often become infected when they are treating patients with suspected or confirmed Ebola virus infection, through direct contact. A total of 558 healthcare workers are known to have been infected in the current outbreak of which 337 have died (data up to 21 November 2014). Furthermore, laboratory accidents and research activities on the virus in human beings and animals may give rise to the risk of infection, when precautions are not rigorously maintained. Individuals remain infected with the virus in their blood and body fluids, including semen and breast milk. After recovery from the disease, men can still transmit the virus through their semen for seven weeks after the disease.

The incubation period for the disease, between penetration of the virus and the start of symptoms, is from two-21 days. The first symptoms are sudden fever and fatigue, muscle pain, headache and sore throat. These symptoms are followed by vomiting, diarrhoea, skin rashes, kidney and liver impairment and, in some cases, internal and external bleeding, such as through the gums and in the faeces. The most frequent laboratory findings are diminished platelet levels and elevation of hepatic enzymes.

In making the diagnosis, it may be difficult to distinguish between infections due to the Ebola virus and due to other diseases such as malaria, typhoid fever and meningoencephalitis. Ebola infection can be confirmed through the following investigations: (i) presence of antibodies seen through ELISA, (ii) detection through antigen uptake, (iii) serological neutralisation test, (iv) electron microscopy and (v) isolation of the virus through culturing. Samples of material from patients are extremely infectious and the laboratory tests have to be performed under the highest safety conditions, both for the technician and for the other people who use the laboratory.

The treatment should consist of support: oral or intravenous rehydration and treatment of the symptoms. There is still no specific treatment, but drugs that have been shown to be specific when used on sick animals are being tested experimentally on humans. Treatment using serum from patients who have been cured of the disease have been promising. Three research projects will take place in West Africa, testing antiviral drugs and the use of survivors' blood to treat the sick. No licensed vaccine is available, but two potential vaccines are being tested on humans.

Prevention and control of the disease is currently based on isolating the patients, absolute protection for the healthcare professionals who are caring for the patients and quarantine for individuals who have come into close contact with patients, including members of their families.

Risk of introduction of the Ebola virus into Brazil - Brazil has a coastal frontier of 7,367 km and its terrestrial limits are its borders with 10 other South American countries (Argentina, Bolivia, Colombia, Guyana, French Guiana, Paraguay, Peru, Suriname, Uruguay and Venezuela) with a total length of the order of $16,886 \mathrm{~km}$. Thus, this is a very extensive terrestrial border along which it is difficult to control the entry of people from other countries. This is particularly so in relation to the countries that border the Brazilian Amazon Region: Bolivia, Colombia, Guyana, French Guiana, Suriname, Peru and Venezuela. On the other hand, more than 25 ports and 20 international airports open up Brazil to the whole world, not only by means of maritime and air transport directly between Brazil and Africa, 
but also particularly from African countries to Europe and other continents and from there to Brazil. According to the World Tourism Organization, in 2012 Brazil received 5.6 million visitors (2014 estimate 6.4 million), however no African country was among the top 20 sending countries.

A study conducted by the Biological System Modeling Laboratory of Northeastern University in the USA, in September 2014, investigated the risk associated with the Ebola outbreak in West Africa in 2014 (Sierra Leone, Guinea, Liberia and Nigeria). The study covered 220 countries and Brazil was in 30th place, with a 5\% risk of importing the virus by 31 October 2014. This represented a high short-term risk. It is to be hoped that this prediction does not become a reality, but preparations need to be made for such an emergency, until such time that the World Health Organization considers that the epidemic in West Africa or in any other country has been brought under control.

TABLE

Outbreaks chronology of Ebola virus disease

\begin{tabular}{|c|c|c|c|c|}
\hline Years/months & Countries/area & $\begin{array}{l}\text { Subtypes } \\
\text { of Ebola }\end{array}$ & $\begin{array}{l}\text { Cases } \\
\text { (n) }\end{array}$ & $\begin{array}{c}\text { Total of death } \\
\mathrm{n}(\%)\end{array}$ \\
\hline 1976 & Congo DRC & Zaire virus & 318 & $280(88)$ \\
\hline 1976 & South Sudan & Sudan virus & 284 & $151(53)$ \\
\hline 1977 & Congo DRC & Zaire virus & 1 & $1(100)$ \\
\hline 1979 & South Sudan & Sudan virus & 34 & $22(65)$ \\
\hline 1994 & Gabon & Zaire virus & 52 & $31(60)$ \\
\hline 1995 & Congo DRC & Zaire virus & 315 & $250(81)$ \\
\hline 1996 January-April & Gabon & Zaire virus & 37 & $21(57)$ \\
\hline 1996 July-1997 January & Gabon & Zaire virus & 60 & $45(74)$ \\
\hline $2000-2001$ & Uganda & Sudan virus & 425 & $224(53)$ \\
\hline 2001 October-2002 March & Gabon & Zaire virus & 65 & $53(82)$ \\
\hline 2001 October-2002 March & Congo DRC & Zaire virus & 57 & $43(75)$ \\
\hline 2002 December-2003 April & Congo DRC & Zaire virus & 143 & $128(89)$ \\
\hline 2003 November-December & Congo DRC & Zaire virus & 35 & $29(83)$ \\
\hline 2004 & South Sudan & Sudan virus & 17 & $7(41)$ \\
\hline 2007 & Congo DRC & Zaire virus & 264 & $187(71)$ \\
\hline 2007 December-2008 January & Uganda & Bundibugyo virus & 149 & $37(25)$ \\
\hline 2008 December-2009 February & Congo DRC & Zaire virus & 32 & $15(47)$ \\
\hline 2011 May & Uganda & Sudan virus & 1 & $1(100)$ \\
\hline 2012 June-October & Uganda & Sudan virus & 11 & $4(36)$ \\
\hline 2012 June-November & Congo DRC & Bundibugyo virus & 36 & $13(36)$ \\
\hline 2012 November-2013 January & Uganda & Sudan virus & 6 & $3(50)$ \\
\hline 2014 August-October & Congo DRC & Zaire virus & 66 & $49(74)$ \\
\hline 2014 March-28 November & Mainly West Africa ${ }^{a}$ & Zaire virus & 15,935 & $5,689(36)$ \\
\hline
\end{tabular}

a: Guinea, Liberia and Sierra Leone have been the most affected countries while cases have been recorded in Nigeria, Senegal, the United States of America (USA), Spain and Mali. Senegal and Nigeria were declared free of Ebola infection by World Health Organization in October 2014. From 1976-2014, about 15 cases of human infection with Ebola virus from monkeys imported cases or laboratory accident of scientists and health workers occurred in USA, England, Italy, South Africa and Russia.

Given the long incubation period of the disease, it is recognised that it is not an easy task to avoid the entry of individuals who are still in the asymptomatic phase or to detect symptomatic individuals at ports, airports and especially at the terrestrial borders, particularly those with countries in the Amazon Region. Nonetheless, in the light of this emergency regarding the possibility of introduction of the Ebola virus into Brazil, integrated surveillance measures are being introduced by the Ministries of Health, Justice and Foreign Relations at Brazil's borders in relation to individuals with possible Ebola virus infection, as follows: (i) detailed review of documentation regarding the origin of passengers arriving at terrestrial, port and airport borders, (ii) establishment of brief questionnaires about countries visited over the preceding 30 days and any manifestations of symptoms that may have resulted from Ebola virus infection, (iii) review of the quarantine law in relation to individuals with suspected Ebola disease, (iv) guidance from the Foreign Ministry for Brazilian citizens who live in countries that are at risk of Ebola disease and for those who come from abroad to Brazil and, lastly, (v) the Ministry of Health has the responsibility for surveillance and medical healthcare for suspected or confirmed cases of this disease. 


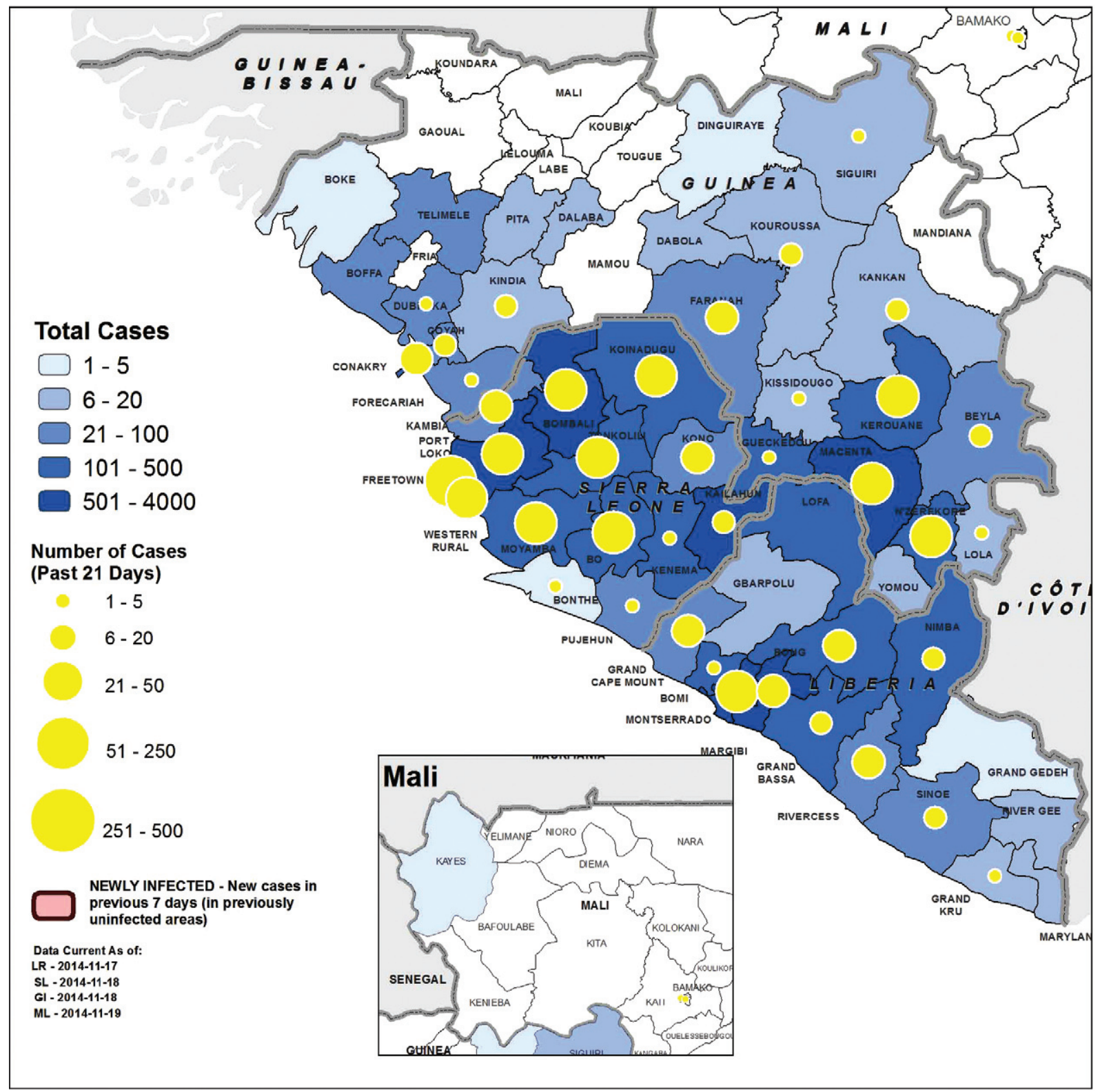

Geographical distribution of probable and confirmed cases in the past 21 days and total cases in Guinea, Liberia, Mali and Sierra Leone. Source: World Health Organization 2014 (dated 21 November). Ebola Response Roadmap Situation Report Update.

In addition to the formal recommendations that the Ministry of Health has been making with regard to surveillance at ports and airports, we suggest that it should make further recommendations to all Brazilian state health departments for preparations for medical care in suspected or confirmed cases of this disease. Given the size of Brazil, it is impossible to predict when the disease might enter the country and it becomes difficult to conduct centralised laboratory tests in Belém, state of Pará (no matter how competent the laboratory is) and to provide medical care at a single hospital in Rio de Janeiro (the current scenario). We suggest to the Ministry of Health to take the following measures: (i) set up a task force of physicians and senior nurses with the capacity to train teams within state health departments, regarding surveillance and medical care for patients with suspected or confirmed Ebola virus infection, (ii) help in setting up and training for diagnostic and medical care centres for this disease, within state health departments and (ii) maintain surveillance at the diagnostic and medical care centres of state health departments through retraining their teams, when necessary. 